\title{
The Hierarchical Operational Space Formulation: Stability Analysis for the Regulation Case
}

\author{
Alexander Dietrich ${ }^{1}$, Christian Ott ${ }^{1}$, and Jaeheung Park ${ }^{2}$
}

\begin{abstract}
The Operational Space Formulation (OSF) from the 1980s is probably the most frequently applied task-space controller in robotics. In multi-priority control of redundant robots via the OSF, a feedback linearization is performed on the first hierarchy level while lower-priority tasks are executed in the dynamically consistent null space of the Jacobian matrices of all higher-priority tasks without disturbing them. However, it has been observed in the past that a formal stability analysis for the overall closed loop is rather difficult, especially for the null space dynamics. Except for exponential stability on the main task level, a complete proof is still missing when the tasks conflict with each other. Here we provide this formal proof of asymptotic stability for the regulation case of a passivity-based OSF controller by means of conditional stability theory and semidefinite Lyapunov functions. Simulations support the intuitive, energy-based interpretation of the proof. This stability analysis lifts the widely used OSF onto a more solid foundation.
\end{abstract}

Index Terms-Compliance and Impedance Control, Redundant Robots, Dynamics

\section{INTRODUCTION}

$\mathbf{T}$ HE control of robots in the task (or operational) space is a standard approach nowadays. Probably the most prominent example is the task definition and execution in Cartesian space of the end-effector of a robotic manipulator. Among these control approaches, the Operational Space Formulation (OSF) [1] plays a central role due to its prevalence in robotics. Therein, a feedback linearization is performed in the task space in order to realize a given, desired dynamics. If a kinematically redundant robot is considered, additional tasks can be simultaneously performed following a hierarchical order. A lower-priority task is then executed in the null space of the Jacobian matrices of all higher-priority tasks [2]-[4], thus it does not affect their performance. If the so-called dynamically consistent null space projector is used, then the dynamics of all hierarchical levels are energetically decoupled due to block-diagonalization of the inertia matrix [5].

Based on this framework numerous modifications, extensions, and practical approaches have been developed since then: from numerically efficient algorithms for the projector computation [6] via appropriate motion generation [7], the

Manuscript received: September, 8, 2017; Revised December, 6, 2017; Accepted December, 29, 2017.

This paper was recommended for publication by Editor Paolo Rocco upon evaluation of the Associate Editor and Reviewers' comments. This work was partially supported by the Bavarian Ministry of Economic Affairs and Media, Energy and Technology, and the project SMiLE.

${ }^{1}$ Alexander Dietrich and Christian Ott are with the Institute of Robotics and Mechatronics, German Aerospace Center (DLR), Wessling, Germany alexander.dietrich@dlr.de

${ }^{2}$ Jaeheung Park is with the Seoul National University

Digital Object Identifier: see top of this page

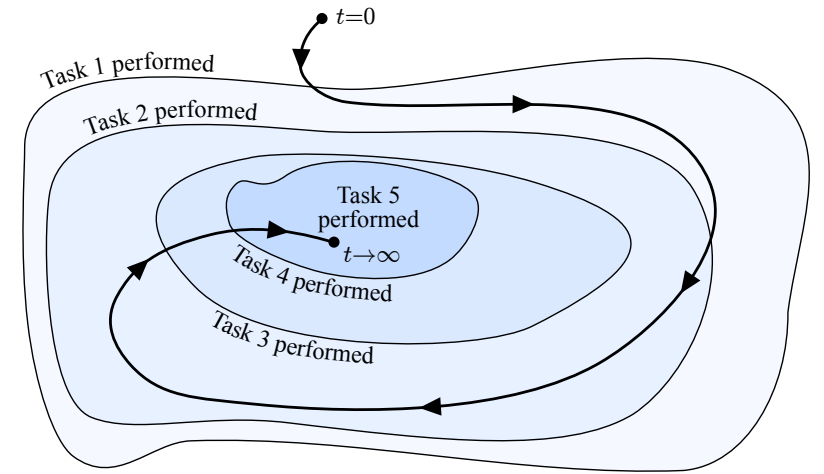

Fig. 1. Graphical interpretation of the convergence stated in the proof of stability. Five hierarchically arranged control tasks in this example are successively performed (as best as possible) for increasing time $(t \rightarrow \infty)$.

incorporation of physical contacts [8] and learning algorithms [9] through to sophisticated control frameworks for humanoid robots [10], [11], lightweight manipulators [12] or intrinsically compliant systems [13]. In fact, even the equality between the classical OSF and common inverse dynamics approaches could be shown [14] recently, which further highlights the importance of the OSF for robotics.

However, the question of stability of the OSF for redundant robots with conflicting tasks is still open. While feedback linearization on the first-priority level provides exponential stability, the properties in the null space remain unclear. The authors in [15] compared different operational space controllers from a theoretical and empirical point of view and summarized that "this difficulty of understanding the null space stability properties is, however, a problem that is shared by all operational space controllers. So far, only empirical evaluations can help to assess the null space robustness".

In the past few years, some works successfully addressed partial aspects of the OSF stability. Nevertheless, an overall proof of stability for the general case has not been provided yet. In [16] asymptotic convergence of a null space velocity error term has been shown in a two-level hierarchy. The same limitation to two priority levels applies to the stability considerations in [17] and [12], where null space compliance was addressed. For multi-priority inverse kinematics, a Lyapunovbased proof has been presented in [18] and conditions for the parameterization of the regulation problem are given. An extended OSF for wheeled humanoid robots has been analyzed in terms of stability in [19] and asymptotic stability of postures could be shown. Based on the theory of conditional stability, we could recently extend the proof of asymptotic stability of the two-level case [17] to an arbitrary number of hierarchy 
levels for the particular case of compliance control [20], [21]. In contrast to the deliberate inertia shaping in the OSF, compliance control requires keeping the natural inertia, which yields configuration-dependencies on all levels. Therefore, exponential stability cannot be shown for the main task, which is a drawback compared with the OSF. Another major difference is that external forces do not have to be fed back to implement the correct impedance when physically interacting with the robot. The control law in the OSF inevitably requires this feedback to realize the desired contact-stiffness behavior.

The main contribution of this paper is the proof of asymptotic stability of the equilibrium for the regulation case of a passivity-based OSF controller with an arbitrary number of priority levels and structural task inconsistencies, that is, not all tasks can be completed simultaneously. In Fig. 2 an example of such a task hierarchy with conflicting objectives is illustrated. We iteratively apply a theorem from conditional stability theory [22], [23] in combination with semi-definite Lyapunov functions [24] in the line of argumentation. In order to conduct the mathematical proof, we express the equations of motion in a task-specific, dynamically decoupled form. Simulations for a multi-priority task hierarchy are performed to interpret the stability properties from a practical point of view. Strictly speaking, the proof implies a successive, hierarchy-consistent task convergence which can be observed in the transients, cf. also Fig. 1.

The paper is organized as follows: After the recapitulation of the fundamentals in Section II, the hierarchical dynamics are derived in Section III. Afterwards, the particular OSF control law is integrated in Section IV and the proof of asymptotic stability is conducted in Section V. Simulations and their interpretation from an energetic point of view are presented in Section VI. After the discussion in Section VII, the paper is concluded in Section VIII.

\section{Fundamentals}

The rigid-body dynamics of a manipulator with $n$ degrees of freedom (DOF) can be written as

$$
\boldsymbol{M}(\boldsymbol{q}) \ddot{\boldsymbol{q}}+\boldsymbol{C}(\boldsymbol{q}, \dot{\boldsymbol{q}}) \dot{\boldsymbol{q}}+\boldsymbol{g}(\boldsymbol{q})=\boldsymbol{\tau}+\boldsymbol{\tau}^{\mathrm{ext}}
$$

with $\boldsymbol{q} \in \mathbb{R}^{n}$ representing the joint configuration. The inertia matrix $\boldsymbol{M}(\boldsymbol{q}) \in \mathbb{R}^{n \times n}$ is symmetric and positive definite, and the generalized gravity forces are represented by $\boldsymbol{g}(\boldsymbol{q})=\left(\partial V_{\mathrm{g}}(\boldsymbol{q}) / \partial \boldsymbol{q}\right)^{T} \in \mathbb{R}^{n}$ with the lower-bounded gravity potential $V_{\mathrm{g}}(\boldsymbol{q}) .^{1}$ The quantities $\boldsymbol{\tau}, \boldsymbol{\tau}^{\mathrm{ext}} \in \mathbb{R}^{n}$ describe the control inputs and generalized external forces, respectively. The Coriolis/centrifugal matrix $\boldsymbol{C}(\boldsymbol{q}, \dot{\boldsymbol{q}}) \in \mathbb{R}^{n \times n}$ complies with $\dot{\boldsymbol{M}}(\boldsymbol{q}, \dot{\boldsymbol{q}})=\boldsymbol{C}(\boldsymbol{q}, \dot{\boldsymbol{q}})+\boldsymbol{C}(\boldsymbol{q}, \dot{\boldsymbol{q}})^{T}$. That relation is equivalent to the skew symmetry of $\dot{\boldsymbol{M}}(\boldsymbol{q}, \dot{\boldsymbol{q}})-2 \boldsymbol{C}(\boldsymbol{q}, \dot{\boldsymbol{q}})$. One can interpret that property as passivity of (1) with respect to the input $\boldsymbol{\tau}+\boldsymbol{\tau}^{\mathrm{ext}}$, the output $\dot{\boldsymbol{q}}$, and the storage function $\frac{1}{2} \dot{\boldsymbol{q}}^{T} \boldsymbol{M}(\boldsymbol{q}) \dot{\boldsymbol{q}}+V_{\mathrm{g}}(\boldsymbol{q})$.

The task hierarchy to be considered involves $r \in \mathbb{N}$ levels. Therefore $r$ task coordinate vectors are introduced which are defined by the mappings $\boldsymbol{x}_{i}=\boldsymbol{f}_{i}(\boldsymbol{q}) \in \mathbb{R}^{m_{i}}$ for $i=1 \ldots r$

\footnotetext{
${ }^{1}$ The gravity potential $V_{\mathrm{g}}(\boldsymbol{q})$ is lower-bounded if purely revolute joints are considered, for example.
}

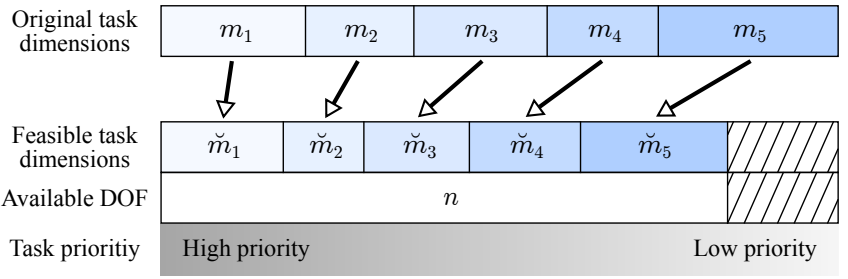

Fig. 2. Task hierarchy on an $n$-DOF robot with five tasks which are in conflict. The subtasks with dimensions $m_{1} \ldots m_{5}$ cannot be completely executed but only partially according to the feasible task dimensions $\breve{m}_{1} \ldots \breve{m}_{5}$.

with the task dimensions $m_{i} \in \mathbb{N}$ and $\sum_{i=1}^{r} m_{i} \geq n$. Generally not all subtasks can be completed, since they are at least partially in conflict with each other. In the order of priority $i=1$ is at the top, and an increasing index stands for a less important task. The five tasks from the example in Fig. 1 are further detailed in Fig. 2, following these definitions. The mappings from joint velocities to task-space velocities are given by the Jacobian matrices $\boldsymbol{J}_{i}(\boldsymbol{q}) \in \mathbb{R}^{m_{i} \times n}$ for $i=1 \ldots r$ :

$$
\dot{\boldsymbol{x}}_{i}=\boldsymbol{J}_{i}(\boldsymbol{q}) \dot{\boldsymbol{q}}, \quad \boldsymbol{J}_{i}(\boldsymbol{q})=\frac{\partial \boldsymbol{f}_{i}(\boldsymbol{q})}{\partial \boldsymbol{q}} .
$$

As of now, the dependencies on the states are omitted in the notations for the sake of readability. Moreover, the so-called augmented Jacobian matrix [4]

$$
\boldsymbol{J}_{i}^{\text {aug }}=\left(\begin{array}{c}
\boldsymbol{J}_{1} \\
\vdots \\
\boldsymbol{J}_{i}
\end{array}\right)
$$

is introduced which takes all tasks "down" to level $i$ into account. The row rank of $\boldsymbol{J}_{r}^{\text {aug }}$ is assumed to be $n$ in the considered workspace, and no singularities are crossed during motions. This aspect will be treated further in the discussion in Section VII. With (2) and (3) one can describe the differential relation for all tasks through

$$
\dot{\boldsymbol{x}}=\boldsymbol{J}_{r}^{\mathrm{aug}} \dot{\boldsymbol{q}} .
$$

where the stacked vector $\boldsymbol{x}=\left(\boldsymbol{x}_{1}^{T}, \ldots, \boldsymbol{x}_{r}^{T}\right)^{T} \in \mathbb{R}^{\sum_{i=1}^{r} m_{i}}$ contains all $r$ task coordinate vectors, and the generalized external forces $\boldsymbol{F}_{\dot{\boldsymbol{x}}_{1}}^{\mathrm{ext}} \ldots \boldsymbol{F}_{\dot{\boldsymbol{x}}_{r}}^{\mathrm{ext}}$ are collocated to the respective task velocities $\dot{\boldsymbol{x}}_{1} \ldots \dot{\boldsymbol{x}}_{r}$. However, since $\dot{\boldsymbol{x}}_{1}$ to $\dot{\boldsymbol{x}}_{r}$ are not dynamically decoupled, they cannot be directly used to implement a dynamically consistent task hierarchy [1]. In this respect, we define new, local (null space) velocities $\boldsymbol{v}_{1}$ to $\boldsymbol{v}_{r}$ following the hierarchy-consistent Jacobian matrices $\breve{\boldsymbol{J}}_{1}$ to $\breve{\boldsymbol{J}}_{r}$.

$$
\underbrace{\left(\begin{array}{c}
\boldsymbol{v}_{1} \\
\vdots \\
\boldsymbol{v}_{r}
\end{array}\right)}_{\boldsymbol{v}}=\underbrace{\left(\begin{array}{c}
\breve{\boldsymbol{J}}_{1} \\
\vdots \\
\breve{\boldsymbol{J}}_{r}
\end{array}\right)}_{\breve{\boldsymbol{J}}} \dot{\boldsymbol{q}}
$$

with the stacked quantities $\boldsymbol{v} \in \mathbb{R}^{n}$ and $\breve{\boldsymbol{J}} \in \mathbb{R}^{n \times n}$. The hierarchy-consistent velocities $\boldsymbol{v}_{i} \in \mathbb{R}^{\breve{m}_{i}}$ and Jacobian matrices $\breve{\boldsymbol{J}}_{i} \in \mathbb{R}^{\breve{m}_{i} \times n}$ for $i=1 \ldots r$ involve the new task dimensions $\breve{m}_{i} \leq m_{i}$. This is due to the possibly conflicting subtasks such that the feasible task dimension on level $i$ reduces from $m_{i}$ to $\breve{m}_{i}$. An intuitive interpretation of the five-level 
example from the introduction is provided in Fig. 2. In order to calculate the new task velocities $\boldsymbol{v}_{i}$ and Jacobian matrices $\breve{\boldsymbol{J}}_{i}$, dynamically consistent [1] null space projectors $\boldsymbol{N}_{i}^{T} \in \mathbb{R}^{n \times n}$ for all hierarchy levels are required, which can be derived through

$$
\boldsymbol{N}_{i}^{T}= \begin{cases}\boldsymbol{I} & \text { if } i=1 \\ \boldsymbol{I}-\boldsymbol{\mathcal { R }}\left(\boldsymbol{J}_{i-1}^{\text {aug }}\right)^{T} \overline{\mathcal{R}}\left(\boldsymbol{J}_{i-1}^{\text {aug }}\right)^{T} & \text { if } 1<i \leq r\end{cases}
$$

with the identity matrix $\boldsymbol{I}$. The bar operator above the variable describes the dynamically consistent pseudoinversion defined by $\overline{\boldsymbol{A}}=\boldsymbol{M}^{-1} \boldsymbol{A}^{T}\left(\boldsymbol{A} \boldsymbol{M}^{-1} \boldsymbol{A}^{T}\right)^{-1}$ for any full-row-rank matrix $\boldsymbol{A} \in \mathbb{R}^{a \times n}$ with $a<n$. The matrix $\boldsymbol{\mathcal { R }}\left(\boldsymbol{J}_{i-1}^{\text {aug }}\right) \in \mathbb{R}^{\sum_{j=1}^{i-1} \breve{m}_{j} \times n}$ describes a basis of the row space of $\boldsymbol{J}_{i-1}^{\text {aug }}$. This term can be derived, for example, via singular value decomposition. The Jacobian matrices can then be obtained via

$$
\breve{\boldsymbol{J}}_{i}=\mathcal{R}\left(\boldsymbol{J}_{i} \boldsymbol{N}_{i}\right)
$$

with the hierarchically consistent task dimension

$$
\breve{m}_{i}=\operatorname{rank}\left(\boldsymbol{J}_{i} \boldsymbol{N}_{i}\right)
$$

Two cases can be distinguished for (8): If $\breve{m}_{i}=m_{i}$ on level $i$, then the respective task can be completed. If $\breve{m}_{i}<m_{i}$, then the task can only partially be performed due to a conflict with a higher-priority objective or a singular Jacobian matrix $\boldsymbol{J}_{i}$ with $\operatorname{rank}\left(\boldsymbol{J}_{i}\right)<m_{i}$.

The generalized external forces $\tau^{\text {ext }}$ can be decomposed into the components $\boldsymbol{F}_{\boldsymbol{v}_{i}}^{\text {ext }} \in \mathbb{R}^{\breve{m}_{i}}$ for $i=1 \ldots r$ collocated to the respective velocities $\boldsymbol{v}_{i}$ via

$$
\left(\begin{array}{c}
\boldsymbol{F}_{\boldsymbol{v}_{1}}^{\mathrm{ext}} \\
\vdots \\
\boldsymbol{F}_{\boldsymbol{v}_{r}}^{\mathrm{ext}}
\end{array}\right)=\breve{\boldsymbol{J}}^{-T} \boldsymbol{\tau}^{\mathrm{ext}}
$$

\section{HIERARCHICAL DYNAMICS}

Using the relations from Section II, we can reformulate the rigid-body dynamics (1) to obtain the decoupled dynamics

$$
\boldsymbol{\Lambda} \dot{\boldsymbol{v}}+\breve{\boldsymbol{C}} \boldsymbol{v}+\breve{\boldsymbol{g}}=\breve{\boldsymbol{J}}^{-T}\left(\boldsymbol{\tau}+\boldsymbol{\tau}^{\mathrm{ext}}\right)
$$

wherein

$$
\begin{aligned}
\breve{\boldsymbol{C}} & =\breve{\boldsymbol{J}}^{-T}\left(\boldsymbol{C}-\boldsymbol{M} \breve{\boldsymbol{J}}^{-1} \dot{\vec{J}}\right) \breve{\boldsymbol{J}}^{-1}, \\
\breve{\boldsymbol{g}} & =\breve{\boldsymbol{J}}^{-T} \boldsymbol{g}, \\
\boldsymbol{\Lambda} & =\breve{\boldsymbol{J}}^{-T} \boldsymbol{M} \breve{\boldsymbol{J}}^{-1}=\operatorname{diag}\left(\boldsymbol{\Lambda}_{1}, \ldots, \boldsymbol{\Lambda}_{r}\right),
\end{aligned}
$$

with the fully coupled Coriolis/centrifugal matrix $\breve{C} \in \mathbb{R}^{n \times n}$, the generalized gravity forces $\breve{g} \in \mathbb{R}^{n}$, and the block-diagonal inertia matrix $\boldsymbol{\Lambda}$. One can derive the decoupled inertias $\boldsymbol{\Lambda}_{i}$ for $i=1 \ldots r$ via $\boldsymbol{\Lambda}_{i}=\left(\breve{\boldsymbol{J}}_{i} \boldsymbol{M}^{-1} \breve{\boldsymbol{J}}_{i}^{T}\right)^{-1}$ [10]. The decomposition of the joint velocity vector $\dot{\boldsymbol{q}}$ into the contributions from all hierarchy levels can be written as

$$
\dot{\boldsymbol{q}}=\sum_{i=1}^{r} \overline{\breve{J}}_{i} \boldsymbol{v}_{i}
$$

This is a consequence of

$$
\breve{\boldsymbol{J}}^{-1}=\left(\overline{\breve{J}}_{1}, \ldots, \overline{\boldsymbol{J}}_{r}\right) .
$$

Moreover, with (15) one can formulate the mapping between the original generalized external forces $\boldsymbol{F}_{\dot{\boldsymbol{x}}_{i}}^{\text {ext }}$ and the corresponding terms $\boldsymbol{F}_{\boldsymbol{v}_{i}}^{\text {ext }}$ in the decoupled space:

$$
\left(\begin{array}{c}
\boldsymbol{F}_{\boldsymbol{v}_{1}}^{\text {ext }} \\
\vdots \\
\boldsymbol{F}_{\boldsymbol{v}_{r}}^{\text {ext }}
\end{array}\right)=\breve{\boldsymbol{J}}^{-T}\left(\boldsymbol{J}_{r}^{\text {aug }}\right)^{T}\left(\begin{array}{c}
\boldsymbol{F}_{\dot{\boldsymbol{x}}_{1}}^{\text {ext }} \\
\vdots \\
\boldsymbol{F}_{\dot{\boldsymbol{x}}_{r}}^{\text {ext }}
\end{array}\right) .
$$

\section{CONTROL DESIGN}

In [15], the most commonly used versions of the OSF are described and analyzed. While many OSF approaches such as [10] suggest the compensation of Coriolis, centrifugal, and gravitational effects on the individual hierarchy levels separately, their level-independent compensation has several advantages as shown in [15]. Therefore, we formulate our particular control law for the regulation case of the OSF as

$$
\boldsymbol{\tau}=\breve{\boldsymbol{J}}^{T}(\breve{\boldsymbol{C}} \boldsymbol{v}+\breve{\boldsymbol{g}})+\boldsymbol{\Psi} \sum_{i=1}^{r}\left(\boldsymbol{J}_{i} \boldsymbol{N}_{i}\right)^{T} \boldsymbol{F}_{i}+(\boldsymbol{\Psi}-\boldsymbol{I}) \boldsymbol{\tau}^{\mathrm{ext}}
$$

$$
\boldsymbol{\Psi}=\breve{\boldsymbol{J}}^{T} \boldsymbol{\Lambda}\left(\boldsymbol{\Lambda}^{\mathrm{des}}\right)^{-1} \breve{\boldsymbol{J}}^{-T} .
$$

The term $\boldsymbol{\Psi} \in \mathbb{R}^{n \times n}$ is used to shape the inertia $\boldsymbol{\Lambda}$ to the desired one $\boldsymbol{\Lambda}^{\text {des }} \in \mathbb{R}^{n \times n}$. Mostly, the choice $\boldsymbol{\Lambda}^{\text {des }}=\boldsymbol{I}$ is made. The generalized control forces $\boldsymbol{F}_{i} \in \mathbb{R}^{m_{i}}$ for all levels $i=1 \ldots r$ implement level-specific desired behaviors, for example via the impedance specification

$$
\boldsymbol{F}_{i}=-\left(\frac{\partial V_{i}\left(\tilde{\boldsymbol{x}}_{i}\right)}{\partial \boldsymbol{x}_{i}}\right)^{T}-\boldsymbol{D}_{i} \dot{\boldsymbol{x}}_{i}
$$

through the gradient of a potential $V_{i}\left(\tilde{\boldsymbol{x}}_{i}\right)$. The quantity $\tilde{\boldsymbol{x}}_{i}=\boldsymbol{x}_{i}-\boldsymbol{x}_{i}^{\text {des }} \in \mathbb{R}^{m_{i}}$ describes the task-space error, that is, the difference between the actual values in the original operational space and the desired ones $\boldsymbol{x}_{i}^{\text {des }}$ (with $\dot{\boldsymbol{x}}_{i}^{\text {des }}=\mathbf{0}$ for the regulation case). A common definition of such a potential is $V_{i}\left(\tilde{\boldsymbol{x}}_{i}\right)=\frac{1}{2} \tilde{\boldsymbol{x}}_{i}^{T} \boldsymbol{K}_{i} \tilde{\boldsymbol{x}}_{i}$ with the positive definite stiffness matrix $\boldsymbol{K}_{i} \in \mathbb{R}^{m_{i} \times m_{i}}$. The active damping in (19) is defined by the positive definite damping matrix $\boldsymbol{D}_{i} \in \mathbb{R}^{m_{i} \times m_{i}}$.

The feedback of the generalized external force $\tau^{\text {ext }}$ in (17) is often omitted in OSF approaches. In fact, if a desired stiffness is intended to be realized for physical interaction, then this feedback ensures the correct contact behavior. Omitting this action would, in turn, inevitably lead to unintuitive, configuration-dependent distortions of the perceived stiffness characteristics.

\section{Proof of Asymptotic Stability}

In this section, the proof of asymptotic stability for the regulation case is presented.

\section{A. Decoupled, level-specific hierarchical dynamics}

With the control law (17) one can reformulate (10) to

$$
\boldsymbol{\Lambda}_{i}^{\mathrm{des}} \dot{\boldsymbol{v}}_{i}+\overline{\boldsymbol{J}}_{i}^{T} \boldsymbol{J}_{i}^{T}\left(\boldsymbol{D}_{i} \dot{\boldsymbol{x}}_{i}+\left(\frac{\partial V_{i}\left(\tilde{\boldsymbol{x}}_{i}\right)}{\partial \boldsymbol{x}_{i}}\right)^{T}\right)=\boldsymbol{F}_{\boldsymbol{v}_{i}}^{\mathrm{ext}}
$$

for all hierarchy levels $i=1 \ldots r$. This beneficial dynamics representation is due to the following relation which results 
from the characteristics of the null space projectors $\boldsymbol{N}_{i}$ while inserting (17):

$$
\breve{\boldsymbol{J}}^{-T} \sum_{i=1}^{r}\left(\boldsymbol{J}_{i} \boldsymbol{N}_{i}\right)^{T} \boldsymbol{F}_{i}=\left(\begin{array}{c}
\overline{\boldsymbol{J}}_{1}^{T} \boldsymbol{J}_{1}^{T} \boldsymbol{F}_{1} \\
\vdots \\
\overline{\boldsymbol{J}}_{r}^{T} \boldsymbol{J}_{r}^{T} \boldsymbol{F}_{r}
\end{array}\right) .
$$

In (21) one can see that the null space projectors cause $\boldsymbol{F}_{i}$ to affect hierarchy level $i$ only. Moreover, one can interpret how $\boldsymbol{F}_{i}$ enters the decoupled dynamics on level $i$ according to (20). Initially $\boldsymbol{F}_{i}$ is mapped to generalized joint forces via $\left(\boldsymbol{J}_{i}\right)^{T}$. Then these generalized joint forces are mapped into the decoupled space through $\left(\overline{\breve{J}}_{i}\right)^{T}$. The dimension changes from $m_{i}$ to $\breve{m}_{i}$, according to the reduction of the dimension of $\dot{\boldsymbol{x}}_{i}$ to the one of $\boldsymbol{v}_{i}$.

The equations of motion (20) impede the conclusion of stability properties. This issue is well known and representative of all null-space-based hierarchical controllers [15].

\section{B. Theorem on conditional stability and strict output passivity}

The proof of asymptotic stability utilizes semi-definite Lyapunov functions in combination with the following theorem on conditional stability to overcome the issues observed in (20).

Theorem 1. [23] Let the system

$$
\begin{aligned}
& \dot{\boldsymbol{z}}=\boldsymbol{a}_{1}(\boldsymbol{z})+\boldsymbol{a}_{2}(\boldsymbol{z}) \boldsymbol{u}, \\
& \boldsymbol{y}=\boldsymbol{b}(\boldsymbol{z})
\end{aligned}
$$

with state $\boldsymbol{z} \in \mathbb{R}^{n}$, input $\boldsymbol{u} \in \mathbb{R}^{m}$, and output $\boldsymbol{y} \in \mathbb{R}^{m}$ be strictly output passive for the output $\boldsymbol{y}=\boldsymbol{b}(\boldsymbol{z})$. Let further $\mathcal{A}$ be the largest positively invariant set contained in $\left\{\boldsymbol{z} \in \mathbb{R}^{n} \mid \boldsymbol{b}(\boldsymbol{z})=\mathbf{0}\right\}$. If the equilibrium $\boldsymbol{z}^{*}$ is asymptotically stable conditionally to $\mathcal{A}$, then it is asymptotically stable for $\boldsymbol{u}=\mathbf{0}$.

For conditional stability, the requirements of common stability definitions must only hold for all those initial conditions which lie in a particular subset. Therefore, conditional stability is weaker than the usual Lyapunov stability. Strict output passivity, as required for Theorem 1, is defined as follows.

Definition 1. [23] A system $\dot{\boldsymbol{z}}=\boldsymbol{f}(\boldsymbol{z}, \boldsymbol{u})$ with input $\boldsymbol{u}$ and output $\boldsymbol{y}$ is said to be strictly output passive if there exists a non-negative function $S(\boldsymbol{z})$ and an $\epsilon>0$ such that

$$
S(\boldsymbol{z}(t))-S(\boldsymbol{z}(0)) \leq \int_{0}^{t}\left(\boldsymbol{y}(s)^{T} \boldsymbol{u}(s)-\epsilon\|\boldsymbol{y}(s)\|^{2}\right) d s
$$

holds for all $t>0$.

\section{Nested sets and storage functions}

As indicated in Theorem 1, subsets will be used within the line of argumentation. The unrestricted, entire state space is defined by $\mathcal{A}_{0}$. In case of free motions $\left(\boldsymbol{F}_{\boldsymbol{v}_{1}}^{\text {ext }}=\mathbf{0}\right)$ the largest positively invariant set for $\left(\boldsymbol{q}, \boldsymbol{v}_{1}=\mathbf{0}, \boldsymbol{v}_{2}, \ldots, \boldsymbol{v}_{r}\right)$ is

$$
\mathcal{A}_{1}=\left\{\left(\boldsymbol{q}, \boldsymbol{v}_{1}, \ldots, \boldsymbol{v}_{r}\right) \mid \boldsymbol{v}_{1}=\mathbf{0}, \boldsymbol{f}_{1}(\boldsymbol{q})=\boldsymbol{x}_{1}^{*}\right\} .
$$

Intuitively, this set contains all system states in which there are no motions on level $(i=1)$ anymore, that is, $\dot{\boldsymbol{v}}_{1}=\boldsymbol{v}_{1}=\mathbf{0}$.

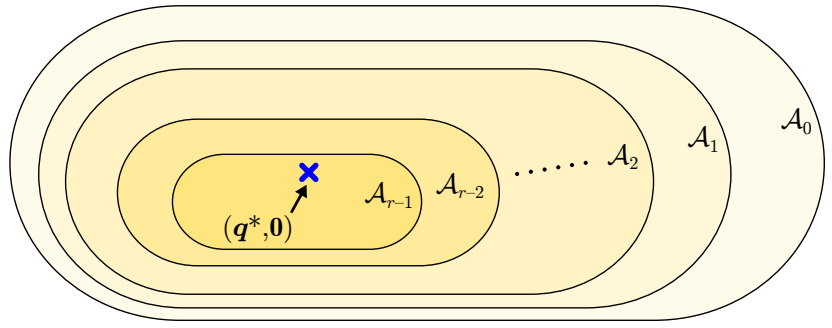

Fig. 3. Location of the hierarchy-consistent, constrained local minimum $\left(\boldsymbol{q}^{*}, \mathbf{0}\right)$ and the nested sets $\mathcal{A}_{0} \ldots \mathcal{A}_{r-1}$ describing the successive, top-down accomplishment of the hierarchically arranged control tasks.

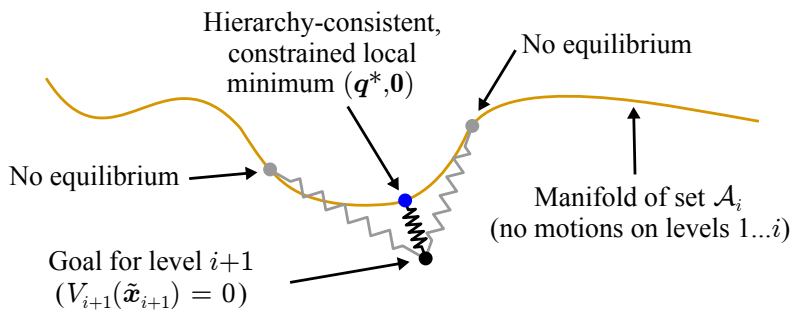

Fig. 4. Graphical interpretation of the equilibrium $\left(\boldsymbol{q}^{*}, \mathbf{0}\right)$

Moreover, the local minimum $\boldsymbol{f}_{1}(\boldsymbol{q})=\boldsymbol{x}_{1}^{*}$ is reached. If the task is completely feasible $\left(\breve{m}_{1}=m_{1}\right)$, then $\boldsymbol{x}_{1}^{*}=\boldsymbol{x}_{1}^{\text {des }}$.

Based on (22) one can recursively define nested sets for the case of free motion with $i=2 \ldots(r-1)$. That describes the additional completion of the subordinate (null space) tasks:

$$
\mathcal{A}_{i}=\mathcal{A}_{i-1} \bigcap\left\{\left(\boldsymbol{q}, \boldsymbol{v}_{1}, \ldots, \boldsymbol{v}_{r}\right) \mid \boldsymbol{v}_{i}=\mathbf{0}, \boldsymbol{f}_{i}(\boldsymbol{q})=\boldsymbol{x}_{i}^{*}\right\} .
$$

Similar to level $i=1$, the operational space coordinates $\boldsymbol{x}_{i}^{*} \in \mathbb{R}^{m_{i}}$ describe either a local minimum for $\breve{m}_{i}<m_{i}$ or the desired values $\boldsymbol{x}_{i}^{\text {des }}$ for $\breve{m}_{i}=m_{i}$. With growing index $i$ in (23) the respective set $\mathcal{A}_{i}$ is successively restricted (compared with its predecessor $\mathcal{A}_{i-1}$ ), until $\mathcal{A}_{r-1}$ is reached, which expresses that all task velocities but the ones on the lowest priority level have already converged to zero. Figure 3 illustrates these nested sets and the hierarchy-consistent, constrained local minimum $\left(\boldsymbol{q}^{*}, \mathbf{0}\right)$, which lies within all sets. In the five-level example of Fig. 1, the equilibrium $\left(\boldsymbol{q}^{*}, \mathbf{0}\right)$ can be interpreted as illustrated in Fig. 4. The lower-priority task (level $i+1)$ cannot be completely executed but only as best as possible within the set $\mathcal{A}_{i}$.

As indicated in Theorem 1 (and further specified in Definition 1) non-negative, state-dependent functions are needed, which we will design from an energetic point of view. On each hierarchy level $i=1 \ldots r$ we establish

$$
S_{i}=\frac{1}{2} \boldsymbol{v}_{i}^{T} \boldsymbol{\Lambda}_{i}^{\mathrm{des}} \boldsymbol{v}_{i}+V_{i}\left(\tilde{\boldsymbol{x}}_{i}\right),
$$

which is positive definite in $\left(\tilde{\boldsymbol{x}}_{i}, \boldsymbol{v}_{i}\right)$ but only positive semidefinite in $(\boldsymbol{q}, \boldsymbol{v})$. Let us consider its time derivative for all hierarchy levels $i=1 \ldots r$ :

$$
\begin{aligned}
\dot{S}_{i}= & \boldsymbol{v}_{i}^{T} \boldsymbol{F}_{\boldsymbol{v}_{i}}^{\mathrm{ext}}-\boldsymbol{v}_{i}^{T} \overline{\breve{\boldsymbol{J}}}_{i}^{T} \boldsymbol{J}_{i}^{T} \boldsymbol{D}_{i} \dot{\boldsymbol{x}}_{i} \\
& -\boldsymbol{v}_{i}^{T} \overline{\boldsymbol{J}}_{i}^{T} \boldsymbol{J}_{i}^{T}\left(\frac{\partial V_{i}}{\partial \boldsymbol{x}_{i}}\right)^{T}+\left(\frac{\partial V_{i}}{\partial \boldsymbol{x}_{i}}\right) \dot{\boldsymbol{x}}_{i}
\end{aligned}
$$


To make (25) easier to interpret one can combine (2) with (14) and introduce the substitution

$$
\dot{\boldsymbol{x}}_{i}=\underbrace{\boldsymbol{J}_{i} \sum_{k=1}^{i-1} \overline{\boldsymbol{J}}_{k} \boldsymbol{v}_{k}}_{\boldsymbol{w}_{i}}+\boldsymbol{J}_{i} \overline{\breve{\boldsymbol{J}}}_{i} \boldsymbol{v}_{i}+\underbrace{\boldsymbol{J}_{i} \sum_{k=i+1}^{r} \overline{\boldsymbol{J}}_{k} \boldsymbol{v}_{k}}_{\mathbf{0}} .
$$

The original task velocity $\dot{\boldsymbol{x}}_{i}$ can be separated into two components: $\boldsymbol{w}_{i} \in \mathbb{R}^{m_{i}}$ describes the part which arises from motions on all higher-priority levels $1 \ldots(i-1)$. The second term $\boldsymbol{J}_{i} \overline{\breve{J}}_{i} \boldsymbol{v}_{i}$ describes the contribution of $\boldsymbol{v}_{i}$ in the original operational space of level $i$. Motions on the lower levels $(i+1) \ldots r$ do not have any influence on $\dot{\boldsymbol{x}}_{i}$ due to the dynamic consistency of the dynamics formulation, since

$$
\boldsymbol{J}_{i} \overline{\breve{J}}_{j}=\mathbf{0} \quad \forall j>i \text {. }
$$

Using (26) one can rewrite (25) to

$$
\dot{S}_{i}=\boldsymbol{v}_{i}^{T} \boldsymbol{F}_{\boldsymbol{v}_{i}}^{\text {ext }}-\boldsymbol{v}_{i}^{T} \breve{\boldsymbol{D}}_{i} \boldsymbol{v}_{i}+\left(\left(\frac{\partial V_{i}}{\partial \boldsymbol{x}_{i}}\right)-\boldsymbol{v}_{i}^{T} \overline{\boldsymbol{J}}_{i}^{T} \boldsymbol{J}_{i}^{T} \boldsymbol{D}_{i}\right) \boldsymbol{w}_{i}
$$

with the positive definite damping matrix

$$
\breve{\boldsymbol{D}}_{i}=\overline{\breve{\boldsymbol{J}}}_{i}^{T} \boldsymbol{J}_{i}^{T} \boldsymbol{D}_{i} \boldsymbol{J}_{i} \overline{\breve{\boldsymbol{J}}}_{i} \text {. }
$$

Eq. (28) clearly reveals a structural issue that impedes the stability analysis: the sign of the term depending on $\boldsymbol{w}_{i}$ is unclear.

Let us assume the case in which a steady state on all higherpriority levels has already been reached, which is given by subset $\mathcal{A}_{i-1}$ implying $\boldsymbol{v}_{k}=\mathbf{0} \forall k<i$, thus $\boldsymbol{w}_{i}=\mathbf{0}$ in (26). As a consequence one obtains

$$
\dot{\boldsymbol{x}}_{i}=\boldsymbol{J}_{i} \overline{\boldsymbol{J}}_{i} \boldsymbol{v}_{i} \quad \forall(\boldsymbol{q}, \boldsymbol{v}) \in \mathcal{A}_{i-1},
$$

which yields the power

$$
\dot{S}_{i}=\boldsymbol{v}_{i}^{T} \boldsymbol{F}_{\boldsymbol{v}_{i}}^{\mathrm{ext}}-\boldsymbol{v}_{i}^{T} \breve{\boldsymbol{D}}_{i} \boldsymbol{v}_{i} \quad \forall(\boldsymbol{q}, \boldsymbol{v}) \in \mathcal{A}_{i-1}
$$

on hierarchy level $i$ indicating strict output passivity for input $\boldsymbol{F}_{\boldsymbol{v}_{i}}^{\text {ext }}$, output $\boldsymbol{v}_{i}$, and the non-negative storage function $S_{i}$ following Definition 1. Note again that this statement is only valid within the set $\mathcal{A}_{i-1}$.

\section{Asymptotic stability of the overall equilibrium}

The proof of asymptotic stability of $\left(\boldsymbol{q}^{*}, \mathbf{0}\right)$ for the regulation case relates to the following proposition.

Proposition 1. Consider the system (10) with feedback law (17)-(19) for the regulation case. The potential functions $V_{i}\left(\tilde{\boldsymbol{x}}_{i}\right)$ for $i=1 \ldots r$ are only positive semi-definite w. r.t. $\boldsymbol{q}$ but positive definite w.r.t. $\tilde{\boldsymbol{x}}_{i}$. The damping matrices $\boldsymbol{D}_{i}$ for $i=1 \ldots r$ are positive definite and the assumption of constant ranks of $\breve{\boldsymbol{J}}_{1} \ldots \breve{\boldsymbol{J}}_{r}$ holds. The subtasks may be inconsistent with each other such that $\boldsymbol{f}_{i}\left(\boldsymbol{q}^{*}\right)=\boldsymbol{x}_{i}^{*}$ for $i=1 \ldots r$ is valid for the equilibrium $(\boldsymbol{q}, \dot{\boldsymbol{q}})=\left(\boldsymbol{q}^{*}, \mathbf{0}\right)$, wherein $\boldsymbol{x}_{i}^{*}$ is not

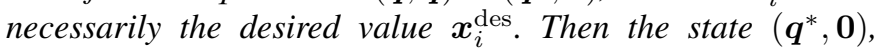
which defines the hierarchy-consistent, local minimum of all $V_{i}\left(\tilde{\boldsymbol{x}}_{i}\right)$, is asymptotically stable for the case of free motion $\left(\boldsymbol{\tau}^{\mathrm{ext}}=\mathbf{0}\right)$.

Proof. The proof of Proposition 1 is conducted in four steps.
1) Stability conditionally to $\mathcal{A}_{r-1}$ : The line of argumentation starts in the subset $\mathcal{A}_{r-1}$, meaning that all task velocities $\boldsymbol{v}_{i}$ are assumed to have already converged to zero except for the ones on the lowest hierarchy level $r$. From (24) and (31) for $i=r$, stability conditionally to $\mathcal{A}_{r-1}$ can be concluded for the case of free motion $\left(\boldsymbol{F}_{\boldsymbol{v}_{r}}^{\text {ext }}=\mathbf{0}\right)$.

2) Asymptotic stability of $\left(\boldsymbol{q}^{*}, \mathbf{0}\right)$ conditionally to $\mathcal{A}_{r-1}$ : According to LaSalle's invariance principle, the state converges to the largest positively invariant set contained in $\mathcal{A}_{r-1}$. The dynamics (20) reveals that this set requires

$$
\overline{\boldsymbol{J}}_{i}^{T} \boldsymbol{J}_{i}^{T}\left(\frac{\partial V_{i}\left(\tilde{\boldsymbol{x}}_{i}\right)}{\partial \boldsymbol{x}_{i}}\right)^{T}=\mathbf{0}
$$

for $\boldsymbol{F}_{\boldsymbol{v}_{i}}^{\text {ext }}=\mathbf{0}$ on all levels $i=1 \ldots r$. This requirement is fulfilled for the hierarchy-consistent, constrained local minimum $\left(\boldsymbol{q}^{*}, \mathbf{0}\right)$, since $\left(\overline{\boldsymbol{J}}_{i}\right)^{T}$ has full row-rank and the applied null space projector $\boldsymbol{N}_{i}^{T}$ ensures static consistency [25]. A statically consistent null space projection means that those components of control actions from lower-priority levels, which are in conflict with any higher-priority levels, do not have an influence in any steady state. This is due to the property $\left(\overline{\breve{J}}_{i}\right)^{T}\left(\boldsymbol{J}_{i-1}^{\text {aug }}\right)^{T}=\mathbf{0}$ as derived in [25], [26]. As a consequence one can conclude asymptotic stability of $\left(\boldsymbol{q}^{*}, \mathbf{0}\right)$ conditionally to $\mathcal{A}_{r-1}$.

3) Application of Theorem 1 within $\mathcal{A}_{r-2}$ : Now Theorem 1 can be applied within the set $\mathcal{A}_{r-2}$ for $\mathcal{A}=\mathcal{A}_{r-1}$, $\boldsymbol{u}=\boldsymbol{F}_{\boldsymbol{v}_{r-1}}^{\text {ext }}$, and $\boldsymbol{y}=\boldsymbol{v}_{r-1}$. The requirement on strict output passivity is satisfied through (31) for $i=r-1$, and asymptotic stability of $\left(\boldsymbol{q}^{*}, \mathbf{0}\right)$ conditionally to $\mathcal{A}_{r-1}$ has already been concluded in the previous step. According to that, asymptotic stability of $\left(\boldsymbol{q}^{*}, \mathbf{0}\right)$ can be deduced. Note again that Theorem 1 has been applied within $\mathcal{A}_{r-2}$ here, thus the asymptotic stability of $\left(\boldsymbol{q}^{*}, \mathbf{0}\right)$ is conditionally to $\mathcal{A}_{r-2}$ only.

4) Iterative application of Theorem 1 (bottom-up across the hierarchy): The previous step can be iteratively repeated, starting within $\mathcal{A}_{r-3}$ for $\mathcal{A}=\mathcal{A}_{r-2}, \boldsymbol{u}=\boldsymbol{F}_{\boldsymbol{v}_{r-2}}^{\text {ext }}$, and $\boldsymbol{y}=\boldsymbol{v}_{r-2}$. The iteration stops when reaching the main task level. There, asymptotic stability of $\left(\boldsymbol{q}^{*}, \mathbf{0}\right)$ conditionally to $\mathcal{A}_{1}$ in combination with the passivity property on level $i=1$ (obtained from (31)) lets us apply Theorem 1 once more and conclude asymptotic stability of $\left(\boldsymbol{q}^{*}, \mathbf{0}\right)$ for the case of free motion.

\section{Simulations}

The energy-based analysis in Section V indicates a successive convergence of the velocities on all priority levels in hierarchical order. In the following, simulations are performed to support the intuitive interpretation of these theoretical findings on a planar, six-DOF robot with conflicting tasks on several hierarchy levels, see Fig. 5. The model contains one prismatic and five revolute joints. Each link is modeled as a bar with a point mass of $1 \mathrm{~kg}$ located in its center. Except for the first link $(0.25 \mathrm{~m})$ all other links have a length of $0.5 \mathrm{~m}$.

The total number of control tasks to be considered is ten. These subtasks are specified in Table I and assigned to five hierarchy levels. The task dimensions change from the initial, original ones $m_{i}$ for $i=1 \ldots 5$ to the hierarchy-consistent, feasible ones $\breve{m}_{i}$ (right column) due to conflicts on three of the 


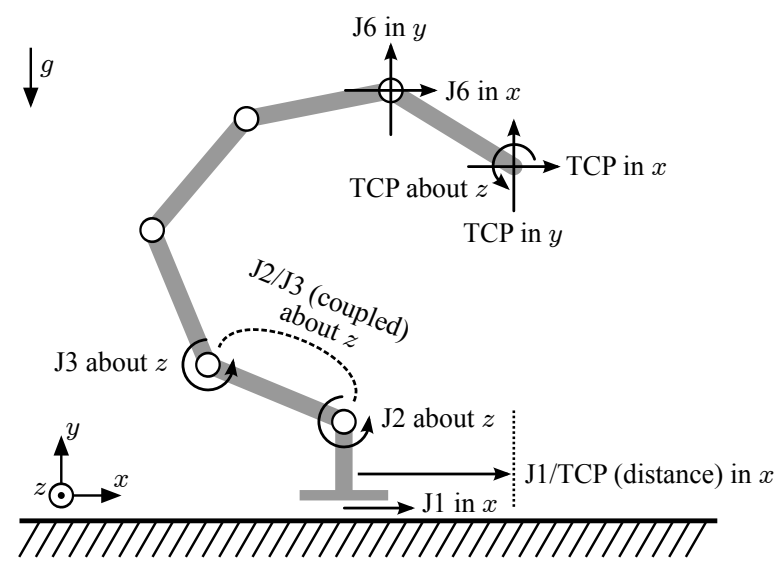

Fig. 5. Simulation model with six DOF. The zero configuration is described by the upright posture. Gravity is simulated with $g=9.81 \frac{\mathrm{m}}{\mathrm{s}^{2}}$. TCP stands for tool center point, and J1 to J6 describe the joints 1 to 6 . Ten control tasks are implemented via impedances, see Table I and Table II for their definitions.

TABLE I

TASK HIERARCHY IN THE SIMULATIONS

\begin{tabular}{|c|l|c|}
\hline Lvl. & Task description & $m_{i} \rightarrow \breve{m}_{i}$ \\
\hline \hline 1 & TCP in $x$, TCP in $y$ & $2 \rightarrow 2$ \\
\hline 2 & J6 in $x$, J6 in $y$ & $2 \rightarrow 1$ \\
\hline 3 & J2 about $z$ & $1 \rightarrow 1$ \\
\hline 4 & J3 about $z$, J2/J3 (coupled) about $z$, TCP about $z$ & $3 \rightarrow 1$ \\
\hline 5 & J1 in $x$, J1/TCP (distance) in $x$ & $2 \rightarrow 1$ \\
\hline
\end{tabular}

five levels. All subtasks are implemented as impedances (19). The corresponding stiffness and damping parameters as well as the initial values and setpoints are provided in Table II. The desired inertias on all hierarchy levels are set to one (in compatible units), that is, $\boldsymbol{\Lambda}^{\text {des }}=\boldsymbol{I}$.

The transients for the regulation case are shown in Fig. 6 by means of the storage functions $S_{i}$ introduced in (24) and their time derivatives $\dot{S}_{i}$ derived in (25). Apparently only the tasks on the levels 1 and 3 can be finished, while they can only be partially completed on the levels 2,4 , and 5 . The hierarchyconsistent, constrained local minimum $(\boldsymbol{q}, \dot{\boldsymbol{q}})=\left(\boldsymbol{q}^{*}, \mathbf{0}\right)$ for $t \rightarrow \infty$ is asymptotically stable as proven in Section V. The errors in the operational spaces of the ten subtasks are depicted in Fig. 7 (left column). In accordance with the energetic characteristics of Fig. 6, the subtasks 2, 4, and 5 can only partially be accomplished.

Furthermore, it should be noted that the task conflicts on the five hierarchy levels are of different nature. While two subtasks compete both on level 2 and on level 5, the case is different for level 4 . There, only the joint impedance $\mathrm{J} 3$ and the coupled impedance $\mathrm{J} 2 / \mathrm{J} 3$ directly compete with each other and yield a local minimum on that very level, whereas the rotational TCP impedance cannot be realized at all. Intuitively said, the tasks on level 1 and level 2 prohibit any further specification of the TCP pose. For this reason, the subtask for the TCP rotation on level 4 has to be canceled, and the corresponding controller gains (stiffness $110 \mathrm{Nm} / \mathrm{rad}$, damping $12 \mathrm{Nms} / \mathrm{rad}$ ) have no impact at all. In Fig. 7, one can also observe the successive
TABLE II

CONTROLLER PARAMETERS (STIFFNESS, DAMPING), INITIAL VALUES IN THE OPERATIONAL SPACE, AND SETPOINTS

\begin{tabular}{|l|l|l|c|c|}
\hline Task & Stiffness & Damping & Initial val. & Setpoint \\
\hline \hline TCP in $x$ & $190 \mathrm{~N} / \mathrm{m}$ & $15 \mathrm{Ns} / \mathrm{m}$ & $0.85 \mathrm{~m}$ & $0.94 \mathrm{~m}$ \\
\hline TCP in $y$ & $190 \mathrm{~N} / \mathrm{m}$ & $15 \mathrm{Ns} / \mathrm{m}$ & $1.10 \mathrm{~m}$ & $0.92 \mathrm{~m}$ \\
\hline \hline $\mathrm{J} 6$ in $x$ & $150 \mathrm{~N} / \mathrm{m}$ & $10 \mathrm{Ns} / \mathrm{m}$ & $0.50 \mathrm{~m}$ & $0.62 \mathrm{~m}$ \\
\hline $\mathrm{J} 6$ in $y$ & $150 \mathrm{~N} / \mathrm{m}$ & $10 \mathrm{Ns} / \mathrm{m}$ & $1.46 \mathrm{~m}$ & $1.35 \mathrm{~m}$ \\
\hline \hline $\mathrm{J} 2$ about $z$ & $180 \mathrm{Nm} / \mathrm{rad}$ & $20 \mathrm{Nms} / \mathrm{rad}$ & $45^{\circ}$ & $45^{\circ}$ \\
\hline \hline $\mathrm{J} 3$ about $z$ & $160 \mathrm{Nm} / \mathrm{rad}$ & $14 \mathrm{Nms} / \mathrm{rad}$ & $-45^{\circ}$ & $-55^{\circ}$ \\
\hline $\mathrm{J} 2 / \mathrm{J} 3$ about $z$ & $160 \mathrm{Nm} / \mathrm{rad}$ & $14 \mathrm{Nms} / \mathrm{rad}$ & $0^{\circ}$ & $-8^{\circ}$ \\
\hline TCP about $z$ & $110 \mathrm{Nm} / \mathrm{rad}$ & $12 \mathrm{Nms} / \mathrm{rad}$ & $-135^{\circ}$ & $-135^{\circ}$ \\
\hline \hline $\mathrm{J} 1$ in $x$ & $100 \mathrm{~N} / \mathrm{m}$ & $10 \mathrm{Ns} / \mathrm{m}$ & $0.00 \mathrm{~m}$ & $-0.05 \mathrm{~m}$ \\
\hline $\mathrm{J} 1 / \mathrm{TCP}$ in $x$ & $100 \mathrm{~N} / \mathrm{m}$ & $10 \mathrm{Ns} / \mathrm{m}$ & $0.85 \mathrm{~m}$ & $0.78 \mathrm{~m}$ \\
\hline
\end{tabular}
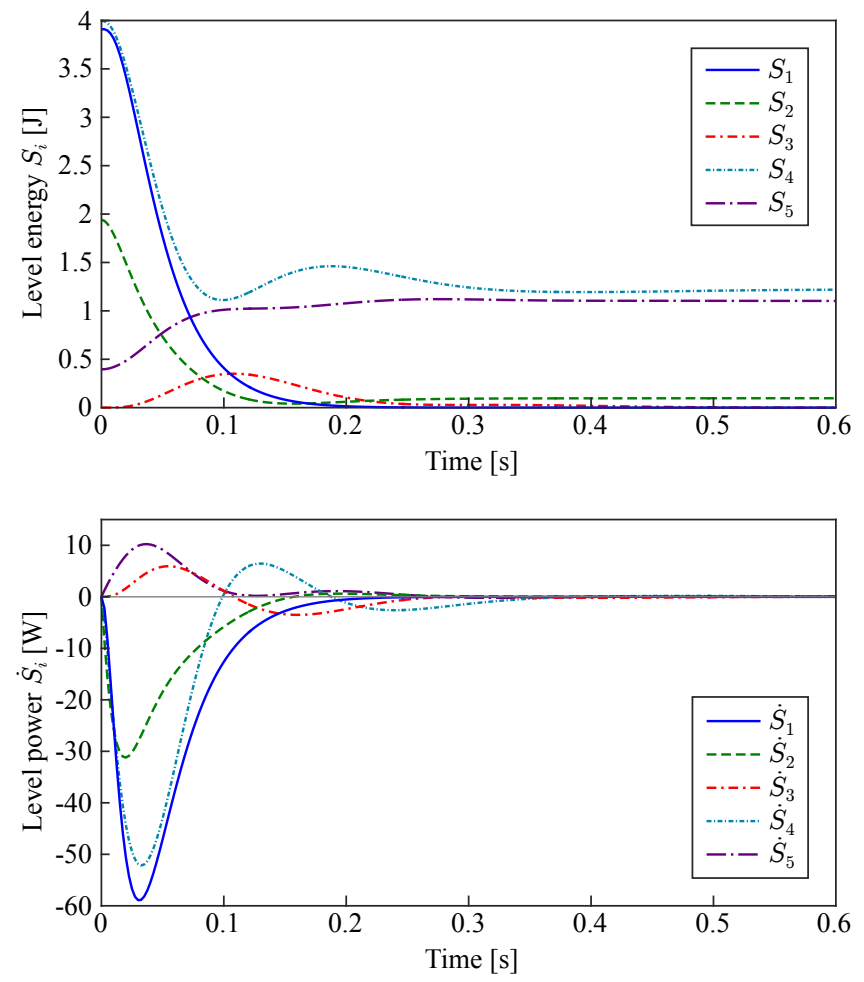

Fig. 6. Level-specific energies $S_{i}$ and powers $\dot{S}_{i}$ for the entire hierarchy with levels $i=1 \ldots 5$, according to (24) and (25).

convergence of the tasks top-down across the hierarchy. This property can be studied by means of the disturbances $\boldsymbol{w}_{i}$ in the diagrams in the right column. The colored arrow indicates this chronological convergence: the lower the priority level, the longer the respective energy $S_{i}$ takes to converge to zero due to the perturbation $\boldsymbol{w}_{i}$, which affects the operational space velocity $\dot{\boldsymbol{x}}_{i}$ as demonstrated in (25) and (26).

\section{DISCUSSION}

The proof of asymptotic stability in Section V can be interpreted as the successive convergence of the hierarchical dynamics following the order of priority. It should be noted that the line of argumentation in the mathematical proof does not demand finite-time convergence on any hierarchy level. 

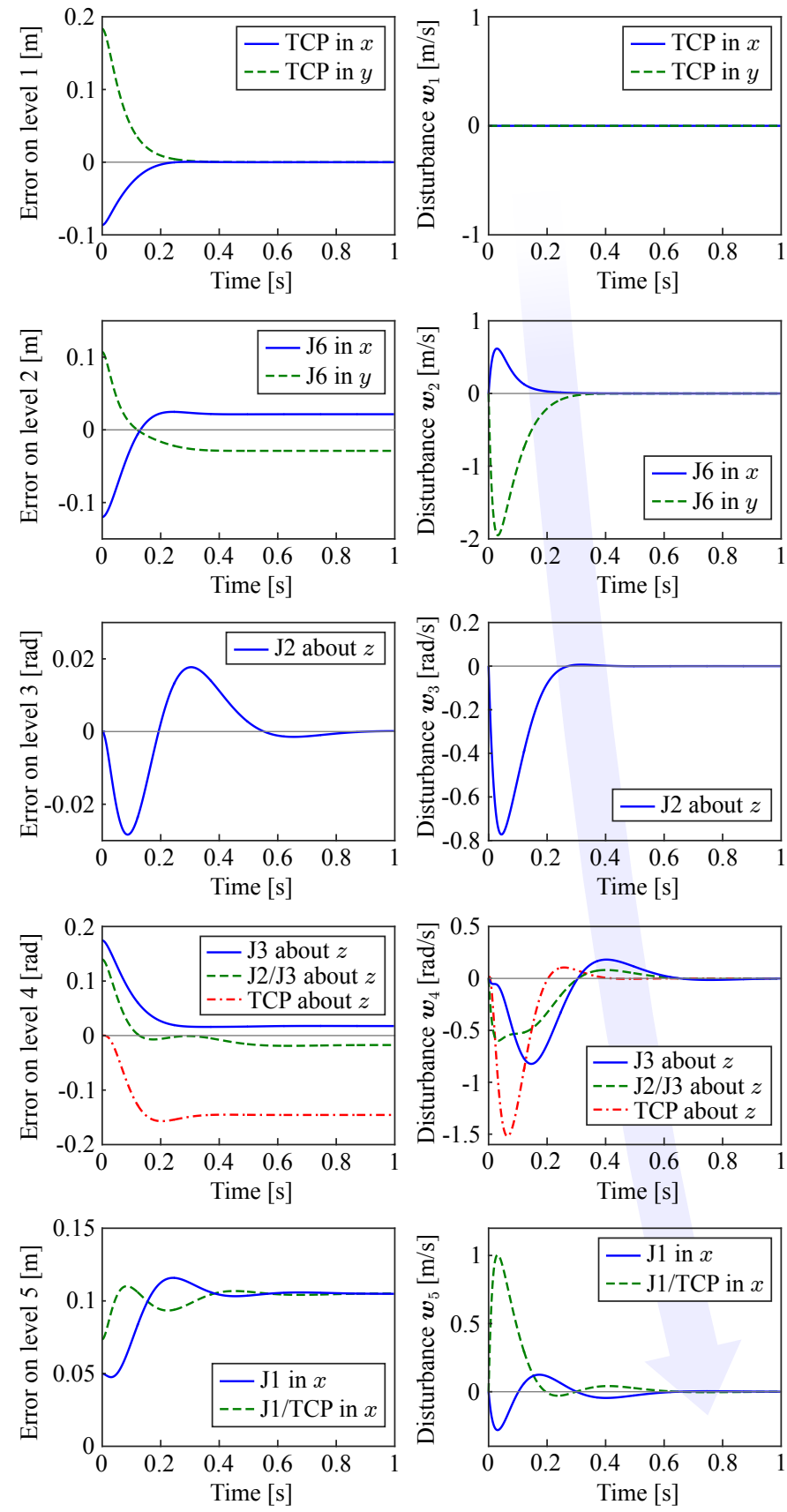

Fig. 7. Errors in the operational space coordinates of all five hierarchy levels (left) and top-down velocity disturbances corresponding to the according priority levels (right). The blue arrow indicates the successive convergence of the disturbances $\boldsymbol{w}_{i}$ for $i=1 \ldots 5$ from (26) to zero.

While this might be counter-intuitive at first glance, it is in fact a fundamental characteristic of conditional stability [22], [23]. Moreover, the used theorem of conditional stability considers the case of free motion. In practice, permanent external forces will yield an equilibrium different from $\left(\boldsymbol{q}^{*}, \mathbf{0}\right)$. This new configuration depends on the stiffness definitions in the impedance specifications (19) of the subtasks.

A benefit of null space projections, as utilized in the OSF, is that task transitions can be handled in general. These cases occur if $\breve{\boldsymbol{J}}_{i}$ changes rank or is replaced by another Jacobian matrix, for example if control tasks are activated/deactivated online or the priority order is modified during task execution. In the literature, a wide variety of singularity-robust approaches have been presented to deal with these issues in kinematic and dynamic control, e.g. [27], [28]. Probably the most known concepts relate to damped least-squares methods [29]. However, the proof of asymptotic stability provided here does not cover such transitions but only considers the times between such switchings. The same applies to singularities which are encountered online, e.g., when crossing a kinematic singularity of a manipulator. Although these scenarios can be handled by the approaches mentioned above, the proof of asymptotic stability does not include the switching/transition process.

Beside the OSF, hierarchical control is often realized by solving numerical optimization problems such as in [30]-[33]. In contrast to the OSF, such approaches make it possible to integrate unilateral and inequality constraints directly (within the formulation of the optimization problem). This is a major drawback of the OSF which can, so far, only be handled by the inclusion of such constraints as classical equality constraints.

The OSF implements the desired task inertia $\boldsymbol{\Lambda}^{\text {des }}$. As shown in (17), this inertia-shaping procedure requires feedback of the generalized external forces $\tau^{\text {ext }}$. It is well known that measuring or estimating these quantities may compromise the robustness. One alternative to inertia shaping is to keep the natural inertia, that is, $\boldsymbol{\Lambda}^{\text {des }}=\boldsymbol{\Lambda}(\boldsymbol{q})$. Then $\boldsymbol{\Psi}$ in (18) reduces to the identity matrix so that (17) does not require feedback of $\tau^{\text {ext }}$ at all. In this so-called compliance control case the dynamics becomes configuration-dependent which must be taken into account when analyzing the stability [20].

The main limitation of the presented proof of asymptotic stability is the restriction to the regulation case and those OSF control approaches which yield a dynamical decoupling as shown in (20). The energy-based analysis of the system controlled via the particular control law (17) builds upon (28), which reveals the detrimental influence of higher-level motions $\boldsymbol{w}_{i}$ on the power $\dot{S}_{i}$ of level $i$. As soon as these motions have stopped, $S_{i}$ decreases monotonically. This property has been utilized by means of the nested sets (22) and (23). However, any higher-level trajectory $\left(\dot{\boldsymbol{x}}_{j}^{\text {des }} \neq \mathbf{0}\right.$ for any $j=1 \ldots(i-1)$ ) results in a permanent top-down disturbance so that the proof is not valid anymore.

Over the years numerous variations of the OSF have been proposed. The classical variation [11], for example, can be applied in the special case of multi-objective control where the subtasks (in combination) exactly occupy all available DOF, i. e. $\sum_{i=1}^{r} m_{i}=n$, and all tasks can actually be completed simultaneously. Then the OSF leads to exponential stability on all hierarchy levels. Since a complete feedback linearization is possible, any dynamics behavior can be imprinted as with inverse dynamics [14]. In contrast to the control law (17)-(19) used here, additional feedback terms are implemented in [11] to avoid the top-down disturbance encountered in (25). While this feedback linearization drastically simplifies the stability analysis, it does, however, not cover the case of task conflicts considered here. 


\section{CONCLUSION}

The Operational Space Formulation (OSF) is a fundamental control concept in kinematically redundant robots and has proven successful in a vast number of implementations in the past decades. However, a formal stability analysis for the OSF with conflicting, hierarchically arranged tasks was lacking so far. Here we have provided this missing link for the regulation case. Based on semi-definite Lyapunov functions and theorems from the field of conditional stability theory we were able to show asymptotic stability of the equilibrium, while the strict task hierarchy was guaranteed throughout. Simulations assisted in the intuitive interpretation of the stability properties, which could be demonstrated from an energetic perspective. We believe that the presented proof of stability lifts the OSF onto a more solid foundation for the future.

\section{REFERENCES}

[1] O. Khatib, "A Unified Approach for Motion and Force Control of Robot Manipulators: The Operational Space Formulation," IEEE Journal of Robotics and Automation, vol. RA-3, no. 1, pp. 43-53, February 1987.

[2] Y. Nakamura, H. Hanafusa, and T. Yoshikawa, "Task-Priority Based Redundancy Control of Robot Manipulators," International Journal of Robotics Research, vol. 6, no. 2, pp. 3-15, June 1987.

[3] J. H. Hollerbach and K. C. Suh, "Redundancy Resolution of Manipulators through Torque Optimization," IEEE Journal of Robotics and Automation, vol. RA-3, no. 4, pp. 308-316, August 1987.

[4] B. Siciliano and J.-J. Slotine, "A General Framework for Managing Multiple Tasks in Highly Redundant Robotic Systems," in Proc. of the 5th International Conference on Advanced Robotics, June 1991, pp. $1211-1216$.

[5] O. Khatib, "Inertial Properties in Robotic Manipulation: An ObjectLevel Framework," International Journal of Robotics Research, vol. 14, no. 1, pp. 19-36, February 1995.

[6] P. Baerlocher and R. Boulic, "Task-Priority Formulations for the Kinematic Control of Highly Redundant Articulated Structures," in Proc. of the 1998 IEEE/RSJ International Conference on Intelligent Robots and Systems, October 1998, pp. 323-329.

[7] O. Brock and O. Khatib, "Elastic Strips: A Framework for Motion Generation in Human Environments," International Journal of Robotics Research, vol. 21, no. 12, pp. 1031-1052, December 2002.

[8] L. Saab, O. E. Ramos, F. Keith, N. Mansard, P. Souères, and J.-I. Fourquet, "Dynamic Whole-Body Motion Generation Under Rigid Contacts and Other Unilateral Constraints," IEEE Transactions on Robotics, vol. 29, no. 2, pp. 346-362, April 2013.

[9] C. Salaün, V. Padois, and O. Sigaud, "Control of redundant robots using learned models: An operational space control approach," in Proc. of the 2009 IEEE/RSJ International Conference on Intelligent Robots and Systems, October 2009, pp. 878-885.

[10] L. Sentis and O. Khatib, "Synthesis of Whole-Body Behaviors through Hierarchical Control of Behavioral Primitives," International Journal of Humanoid Robotics, vol. 2, no. 4, pp. 505-518, January 2005.

[11] O. Khatib, L. Sentis, J. Park, and J. Warren, "Whole-Body Dynamic Behavior and Control of Human-like Robots," International Journal of Humanoid Robotics, vol. 1, no. 1, pp. 29-43, March 2004.

[12] H. Sadeghian, L. Villani, M. Keshmiri, and B. Siciliano, "Task-Space Control of Robot Manipulators With Null-Space Compliance," IEEE Transactions on Robotics, vol. 30, no. 2, pp. 493-506, April 2014.

[13] M. Hutter, M. Hoepflinger, C. Gehring, M. Bloesch, C. D. Remy, and R. Siegwart, "Hybrid Operational Space Control for Compliant Legged Systems," in Proc. of the 8th Robotics: Science and Systems Conference, 2012.

[14] L. Righetti, J. Buchli, M. Mistry, and S. Schaal, "Inverse Dynamics Control of Floating-Base Robots with External Constraints: a Unified View," in Proc. of the 2011 IEEE International Conference on Robotics and Automation, May 2011, pp. 1085-1090.
[15] J. Nakanishi, R. Cory, M. Mistry, J. Peters, and S. Schaal, "Operational Space Control: A Theoretical and Empirical Comparison," International Journal of Robotics Research, vol. 27, no. 6, pp. 737-757, June 2008.

[16] C. Natale, B. Siciliano, and L. Villani, "Spatial Impedance Control of Redundant Manipulators," in Proc. of the 1999 IEEE International Conference on Robotics and Automation, May 1999, pp. 1788-1793.

[17] C. Ott, A. Kugi, and Y. Nakamura, "Resolving the Problem of Nonintegrability of Nullspace Velocities for Compliance Control of Redundant Manipulators by using Semi-definite Lyapunov functions," in Proc. of the 2008 IEEE International Conference on Robotics and Automation, May 2008, pp. 1999-2004.

[18] G. Antonelli, "Stability Analysis for Prioritized Closed-Loop Inverse Kinematic Algorithms for Redundant Robotic Systems," IEEE Transactions on Robotics, vol. 25, no. 5, pp. 985-994, October 2009.

[19] L. Sentis, J. Petersen, and R. Philippsen, "Implementation and stability analysis of prioritized whole-body compliant controllers on a wheeled humanoid robot in uneven terrains," Autonomous Robots, vol. 35, no. 4, pp. 301-319, 2013.

[20] A. Dietrich, C. Ott, and A. Albu-Schäffer, "Multi-Objective Compliance Control of Redundant Manipulators: Hierarchy, Control, and Stability," in Proc. of the 2013 IEEE/RSJ International Conference on Intelligent Robots and Systems, November 2013, pp. 3043-3050.

[21] C. Ott, A. Dietrich, and A. Albu-Schäffer, "Prioritized Multi-Task Compliance Control of Redundant Manipulators," Automatica, vol. 53, pp. 416-423, March 2015.

[22] R. Sepulchre, M. Jankovic, and P. Kokotovic, Constructive Nonlinear Control. Springer, 1997.

[23] A. van der Schaft, $L_{2}$-Gain and Passivity Techniques in Nonlinear Control, 2nd ed. Springer Publishing Company, Berlin Heidelberg, 2000.

[24] A. Iggidr, B. Kalitine, and R. Outbib, "Semidefinite Lyapunov Functions Stability and Stabilization," Mathematics of Control, Signals, and Systems, vol. 9, no. 2, pp. 95-106, 1996.

[25] A. Dietrich, Whole-Body Impedance Control of Wheeled Humanoid Robots, ser. Springer Tracts in Advanced Robotics. Springer International Publishing, 2016, vol. 116.

[26] A. Dietrich, C. Ott, and A. Albu-Schäffer, "An overview of null space projections for redundant, torque-controlled robots," International Journal of Robotics Research, vol. 34, no. 11, pp. 1385-1400, Sept. 2015.

[27] J. Lee, N. Mansard, and J. Park, "Intermediate Desired Value Approach for Task Transition of Robots in Kinematic Control," IEEE Transactions on Robotics, vol. 28, no. 6, pp. 1260-1277, December 2012.

[28] A. Dietrich, A. Albu-Schäffer, and G. Hirzinger, "On Continuous Null Space Projections for Torque-Based, Hierarchical, Multi-Objective Manipulation," in Proc. of the 2012 IEEE International Conference on Robotics and Automation, May 2012, pp. 2978-2985.

[29] A. Deo and I. Walker, "Overview of Damped Least-Squares Methods for Inverse Kinematics of Robot Manipulators," Journal of Intelligent Robotic Systems, vol. 14, no. 1, pp. 43-68, September 1995.

[30] O. Kanoun, F. Lamiraux, and P.-B. Wieber, "Kinematic Control of Redundant Manipulators: Generalizing the Task-Priority Framework to Inequality Task," IEEE Transactions on Robotics, vol. 27, no. 4, pp. 785-792, August 2011.

[31] A. Escande, N. Mansard, and P.-B. Wieber, "Hierarchical quadratic programming: Fast online humanoid-robot motion generation," International Journal of Robotics Research, vol. 33, no. 7, pp. 1006-1028, June 2014.

[32] A. Herzog, L. Righetti, F. Grimminger, P. Pastor, and S. Schaal, "Balancing experiments on a torque-controlled humanoid with hierarchical inverse dynamics," in Proc. of the 2014 IEEE/RSJ International Conference on Intelligent Robots and Systems, September 2014, pp. 981-988.

[33] D. J. Agravante, G. Claudio, F. Spindler, and F. Chaumette, "Visual Servoing in an Optimization Framework for the Whole-Body Control of Humanoid Robots," IEEE Robotics and Automation Letters, vol. 2. no. 2, pp. 608-615, April 2017. 